\title{
Local Properties of an Isothermal Charged Fluid: Initial-Boundary Value Problem *
}

\author{
Joseph W. Jerome ${ }^{\mathrm{a}}$ \\ ${ }^{a}$ Department of Mathematics, Northwestern University, \\ Evanston, IL 60208-2730, USA
}

\begin{abstract}
The Cauchy problem for the one-dimensional isothermal Euler-Poisson system was investigated by F. Poupaud, M. Rascle, and J.-P. Vila in [J. Diff. Equations 123 (1995), 93-121]. Glimm's scheme was employed to obtain a global entropic solution. It appears that the initial-boundary value problem has not been investigated previously for this system, except in the isentropic case, via an approach based on compensated compactness. While the isothermal case, employing the ideal gas law for the pressure, suggests artificial diffusion/viscosity, the underlying infrastructure (the analog of Glimm's scheme) has not yet been established to analyze the initialboundary value problem. We begin a program here, utilizing diffusion and viscosity. By employing Kato's theory of evolution operators, we provide a local smooth existence/uniqueness theory. A theorem of Smoller, as generalized by Fang and Ito [Nonlinear Anal. 28 (1997), 947-963], is used to obtain invariant region bounds for the evolution. Because the theory is local, shocks do not appear, either in the parabolic system, or its vanishing viscosity limit.
\end{abstract}

\section{Introduction}

The isothermal Euler-Poisson gas was studied by F. Poupaud, M. Rascle, and J.-P. Vila in [8], where a global entropic solution to the Cauchy problem in one dimension was established by Glimm's method [3]. Noteworthy aspects include the non-vacuum assumption on the initial concentration, which is maintained in the present study. Here, we are concerned with the initial-boundary value problem for this system, and we suggest a vanishing viscosity method. The boundary conditions are discussed later in the introduction.

* Dedicated to Professor V. Lakshmikantham on the occasion of his eighty-fifth birthday in recognition of his exceptional scholarly accomplishments and service 
The isothermal model uses the ideal gas law as the pressure constitutive relation. This is consistent with modeling of transport in semiconductors and ion channels [6]. It excludes, however, approaches based on compensated compactness (cf. $[2,1]$ ) where the so-called isentropic model is employed, via the adiabatic pressure relation.

\subsection{The Isothermal Euler-Poisson System}

We present here the equations of the isothermal Euler-Poisson gas.

$$
\begin{gathered}
n_{t}+(n v)_{x}=0 \quad \text { Conservation of particles } \\
p_{t}+\left(p v+n k T_{0}\right)_{x}=-e n E-p / \tau_{p} \quad \text { Conservation of momentum }
\end{gathered}
$$

The electric field $E=-\Phi_{x}$ is determined by

$$
\epsilon_{0} \Phi_{x x}=e\left(n+n_{D}\right) \quad \text { Poisson equation, }
$$

where we use the subscripts $t, x$ for derivatives with respect to $t, x$. The fundamental dependent variables are $n, p, \Phi$. For simplicity, the spatial interval is selected as $[0,1]$. The above symbols are defined as follows:

$\epsilon_{0}$ is the dielectric constant of the device; $n_{D}$ is the (smooth) doping; $n$ is the electron concentration; $v$ is the electron translational velocity; $p$ is the electron momentum density, $p=m n v ; m$ is the mass of the electron; $k$ is Boltzmann's constant; $e$ is the electron charge; $\Phi$ is the electric potential as a function

of position; $\tau_{p}$ is the momentum relaxation time of the electrons; $T_{0}$ is the constant electron temperature.

\section{Boundary Conditions}

Homogeneous Dirichlet boundary conditions are specified for $p$, and homogeneous Neumann boundary conditions are specified for $\Phi$.

\section{Initial Conditions}

Initial conditions are specified for $n, p$ at time $t=0$, with a no vacuum assumption for $n$. Regularity is specified by the space $Y$ of the sequel.

\subsection{Introduction of Diffusion and Viscosity Parameters}

We present here the modified equations. Previous boundary conditions are augmented by homogeneous Neumann boundary conditions for $n$. 


$$
\begin{gathered}
n_{t}+(n v)_{x}=\varepsilon n_{x x} \quad \text { Artificial diffusion } \\
p_{t}+\left(p v+n k T_{0}\right)_{x}=-e n E-p / \tau_{p}+\varepsilon p_{x x} \quad \text { Artificial viscosity. }
\end{gathered}
$$

The electric field $E=-\Phi_{x}$ is determined by the equation (3).

\section{A Stable Method for Smooth Solutions of the Viscosity System}

We outline the approach to the parabolic system, based on evolution operators. It will follow the following lines: (i) reformulation in symmetrizable format; (ii) a general summary of the abstract theory; (iii) its application to the parabolic system. Solutions remain stable as $\varepsilon \rightarrow 0$.

\subsection{System Reformulation in Nonconservative Form}

We reformulate the system $(4,5)$ in nonconservative form via the dependent variables $n$ and $v$. As always, there is coupling to (3). Elementary differential calculus gives the equivalent system:

$$
\begin{aligned}
n_{t}+v n_{x}+n v_{x} & =\varepsilon n_{x x}, \\
v_{t}+v v_{x}+\frac{k T_{0}}{m n} n_{x}-\frac{2 \varepsilon n_{x} v_{x}}{n} & =\varepsilon v_{x x}-\frac{e}{m} E-\frac{v}{\tau_{p}} .
\end{aligned}
$$

Define the 2-vector $\mathbf{u}$ and the system symmetrizer $A_{0}(\mathbf{u})$ by

$$
\mathbf{u}=\left[\begin{array}{l}
n \\
v
\end{array}\right], \quad A_{0}(\mathbf{u})=\left[\begin{array}{c|c}
\frac{k T_{0}}{m n} & 0 \\
\hline 0 & n
\end{array}\right]
$$

Upon factorization, the system may be written in matrix format:

$$
\frac{d \mathbf{u}}{d t}+A_{0}^{-1} A_{1} \frac{d \mathbf{u}}{d x}=\varepsilon \frac{d^{2} \mathbf{u}}{d x^{2}}+F(t, \mathbf{u}(t))
$$

where $A_{1}$ is given by the symmetric matrix:

$$
A_{1}(\mathbf{u})=\left[\begin{array}{c|c}
\frac{c_{0} v}{n} & c_{0} \\
\hline c_{0} & n v-2 \varepsilon n_{x}
\end{array}\right],
$$

with $c_{0}=k T_{0} / m$. $F$ is defined below, and incorporates the forcing terms. 


\subsection{The Abstract Cauchy Problem}

Consider the Cauchy problem:

$$
\frac{d u}{d t}+A(t, u) u=F(t, u), \quad 0 \leq t \leq T, \quad u(0)=u_{0}
$$

where the unknown $u$ takes values in a Banach space and $A(t, u)$ is a linear operator depending on $t$ and $u$. A concise framework, due to Kato, is introduced to define and analyze this problem. This theory is outlined in [5]. We begin with some familiar terminology.

Definition 2.1 Let $U$ be a closed linear operator with domain and range dense in a Banach space $X$. Denote by $R(\lambda, U)$ the resolvent $(\lambda I-U)^{-1}$ for $\lambda$ in the resolvent set of $U$. For $M>0$ and $\omega \in R$ denote by $G(X, M, \omega)$ the set of all operators $A=-U$ such that

$$
\left\|[R(\lambda, U)]^{r}\right\| \leq M(\lambda-\omega)^{-r}, r \geq 1, \lambda>\omega
$$

Finally,

$$
G(X)=\cup_{\omega, M} G(X, M, \omega)
$$

The operators $U$ are generators of strongly continuous semigroups on $X$. This property extends to smooth spaces $Y$ embedded in $X$. We extract now the basic hypotheses required for an abstract existence/uniqueness theory. For this, we follow [5, Chaps. 6,7].

\section{Topology}

$Y, X$ and $Z$ are reflexive Banach spaces satisfying

$$
Y \subset X \subset Z
$$

where the inclusions are dense and continuous; $W$ is an open subset of $Y$. If $N(Z)$ denotes the metric space of norms equivalent to $\|\cdot\|_{Z}$, with metric

$$
d\left(\|\cdot\|_{\mu},\|\cdot\|_{\nu}\right)=\ln \max \left\{\sup _{z \neq 0} \frac{\|z\|_{\mu}}{\|z\|_{\nu}}, \sup _{z \neq 0} \frac{\|z\|_{\nu}}{\|z\|_{\mu}}\right\},
$$

a subfamily $\{N(t, w)\}$ exists for each pair $(t, w) \in[0, T] \times W$, satisfying:

$$
\begin{aligned}
d\left(N(t, w),\|\cdot\|_{Z}\right) & \leq \lambda_{N}, \\
d\left(N\left(t^{\prime}, w^{\prime}\right), N(t, w)\right) & \leq \mu_{N}\left(\left|t^{\prime}-t\right|+\left\|w^{\prime}-w\right\|_{X}\right) .
\end{aligned}
$$




\section{Generator Properties}

Assume $A(t, w) \in G\left(Z_{N(t, w)}, 1, \omega\right), \forall w \in W, \forall 0 \leq t \leq T$. Furthermore, the mapping $t \mapsto A(t, w) \in B[Y, X]$ is continuous in norm for each $w$, with $\|A(t, w)\|_{Y, X} \leq \lambda_{A}$ and

$$
\|A(t, u)-A(t, v)\|_{Y, X} \leq \mu_{A}\|u-v\|_{X}
$$

It is assumed that $Y$ is a subset of the domain of each generator.

\section{Hypotheses on $F$}

$t \mapsto F(t, w)$ is required to be strongly continuous into $X$ for each $w$; the image $F(t, w)$ is required to be in $Y$ with $\|F(t, w)\|_{Y} \leq \lambda_{F}$. Furthermore,

$$
\|F(t, u)-F(t, v)\|_{X} \leq \mu_{F}\|u-v\|_{X}
$$

\section{Isomorphism}

There is an isomorphism, denoted $S: Y \mapsto Z$, satisfying, for each $A(t, w)$,

$$
S A(t, w) S^{-1}=A(t, w)+B(t, w)
$$

where $B(t, w)$ is a bounded linear operator on $Z$, with $\|B(t, w)\|_{Z} \leq \lambda_{B}$.

The following result was proved in [4] (also found in [5]).

Proposition 2.1 Under the hypotheses (1-4) just stated, and the hypothesis $u_{0} \in W$, there is a time $T^{\prime} \leq T$ and a unique $u \in C\left(\left[0, T^{\prime}\right] ; W\right)$, with $\frac{d u}{d t} \in$ $C\left(\left[0, T^{\prime}\right] ; X\right)$, satisfying the Cauchy problem (11).

\subsection{Application of the Theory to the Viscosity System}

We will make the proper identifications with the preceding abstract result. We begin with the topological identifications. Define

$$
\begin{aligned}
& Z:=L_{2}(0,1) \otimes L_{2}(0,1) \\
& X:=H^{2}(0,1) \otimes H^{2}(0,1), \\
& \mathbf{D}=\left\{f \in H^{2}(0,1): f^{\prime}(0)=f^{\prime}(1)=0\right\} \otimes\left\{f \in H^{2}(0,1): f(0)=f(1)=0\right\} \\
& Y \subset \mathbf{D} \cap\left(H^{s}(0,1) \otimes H^{s}(0,1)\right), s>5 / 2 .
\end{aligned}
$$


Here, the precise specification of $Y$ will be made below in the course of the definition of the isomorphism. If initial data $n_{0}, v_{0}$ are given, set

$$
\mathbf{u}_{0}=\left[\begin{array}{l}
n_{0} \\
v_{0}
\end{array}\right],
$$

and suppose that the pointwise inequality $n_{0}(x) \geq \alpha>0$ holds uniformly on $[0,1]$. Because the symmetrizer experiences vacuum singularities, we regularize $A_{0}$ as follows. If $0<\alpha^{\prime}<\alpha$, and $\zeta(r)$ is a non-decreasing $C^{\infty}$ function which vanishes for $r \leq 0$ and is unity for $r \geq 1$, define the regularized symmetrizer $\tilde{A}_{0}$ by the replacement:

$$
n \mapsto(1 / 2) n\left(1+\zeta\left(n / \alpha^{\prime}\right)\right)
$$

in the diagonal positions; $\tilde{A}_{0}$ agrees with $A_{0}$ if $n \geq \alpha^{\prime}$. We identify the set $W$ of the previous proposition with a ball containing the initial datum $\mathbf{u}_{0}$ (radius $r$ arbitrarily specified in advance). Finally, the equivalent norms required for the generators are given by:

$$
\|f\|_{N(\mathbf{w})}=\left\|\tilde{A}_{0}(\mathbf{w}) f\right\|_{Z} .
$$

Note the absence of direct dependence on $t$. In fact, in the interpretation of (11), the operators $A(t, \mathbf{u})$ do not depend explicitly upon $t$. We now discuss the generators. The domains of these operators $A(\mathbf{u})$ are invariant: $\mathbf{D}$. The operator definitions are given by:

$$
A(\mathbf{u}) \mathbf{w}=\tilde{A}_{0}^{-1}(\mathbf{u}) A_{1}(\mathbf{u}) \frac{d \mathbf{w}}{d x}-\frac{\varepsilon d^{2} \mathbf{w}}{d x^{2}} .
$$

Note that we use here the definitions of $(8,10)$.

In order to define the isomorphism $S$, we proceed as follows. It is known that

$$
\Gamma^{s}=\left(I-d^{2} / d x^{2}\right)^{s / 2}
$$

is an isomorphism from $H^{s}(\mathbf{R})$ to $L_{2}(\mathbf{R})$. We now define $S^{-1}$ and, simultaneously, $Y$. It is more convenient to consider the individual components of the mapping. Thus, for the first component, let $f \in L_{2}(0,1)$, and denote by $\tilde{f}$ its extension by zero to $\mathbf{R}$. Set $g=\Gamma^{-s} \tilde{f}$, and $w=\Gamma^{2} g$. Denote by $u$ the unique solution of the two-point boundary value problem on $[0,1]$ :

$$
\Gamma^{2} u=w, u^{\prime}(0)=u^{\prime}(1)=0
$$

We have defined the first component $u$ of $S^{-1}$. The second is analogously defined, via the boundary-value problem:

$$
\Gamma^{2} u=w, u(0)=u(1)=0 .
$$


This defines $S^{-1}$, and we set $Y=S^{-1}\left(L_{2}(0,1) \otimes L_{2}(0,1)\right)$. Note that $Y$ is a Hilbert subspace as described in (16). We assert that $S$ is an isomorphism of $Y$ onto $L_{2}(0,1) \otimes L_{2}(0,1)$. It suffices to prove that $S^{-1}$ is injective. Suppose that $u=S^{-1} f_{1}$ and $u=S^{-1} f_{2}$. By maintaining the above notation, one infers that $\Gamma^{2} \otimes \Gamma^{2} g_{1}$ agrees with $\Gamma^{2} \otimes \Gamma^{2} g_{2}$ on $[0,1]$. One argues, via elementary solutions, that $g_{1}-g_{2}$ is in the $L_{2}(0,1) \otimes L_{2}(0,1)$ - null space of $\Gamma^{2} \otimes \Gamma^{2}$, hence is the zero element. It follows that $\tilde{f}_{1}=\tilde{f}_{2}$, so that $f_{1}=f_{2}$.

The definition of $F$ incorporates the nonlocal Poisson solver map $\Phi$ :

$$
F(t, \mathbf{u})=\left[\begin{array}{c}
0 \\
-\frac{e}{m}\left(\frac{-d \Phi(\mathbf{u})}{d x}\right)-\frac{v}{\tau_{p}}
\end{array}\right]
$$

Note that $F(t, \mathbf{u}) \in Y$ for each $t$.

Theorem 2.1 Under the hypotheses stated above, including $s>5 / 2$, there exists a $T>0$ such that a unique solution $\mathbf{u}$ of the system $(6,7,3)$ (equivalently, $(4,5,3))$ exists on $[0, T]$. The system satisfies the initial condition and is in the regularity class specified in the general theorem:

$$
u \in C([0, T] ; W), \frac{d u}{d t} \in C([0, T] ; X) .
$$

$W$ is any open set containing the initial data, and the time evolution can be constrained to remain in $W$. Note that $W$ does not depend on $\varepsilon$. In particular, the solutions converge to the conservation law solution of $(1,2,3)$ as $\varepsilon \rightarrow 0$. The invariant region estimates to follow remain stable under $\varepsilon \rightarrow 0$.

Proof We infer from the Friedrich's inequality and the perturbation theory of generators that $A(\mathbf{w}) \in G\left(Z_{N(\mathbf{w})}, 1, \omega\right)$. Specifically, both the second order operator term and the first order operator term are separately in $G\left(Z_{N(\mathbf{w})}, 1, \omega\right)$. The sum is stable with respect to the second argument because of relative boundedness. Here, $\omega$ may be estimated in terms of the $C^{1}$ norm of the entries of $A_{0}$ and $A_{1}$, which leads to a functional form: $\omega=\omega(r)$, not depending upon $\varepsilon$.

The Lipschitz property (14) requires the introduction of the space $X$ intermediate between $Y$ and $Z$. If we use the notation,

$$
E(\mathbf{u})=\tilde{A}_{0}^{-1}(\mathbf{u}) A_{1}(\mathbf{u}(t)) \partial / \partial x
$$

then it is seen that the mapping $\mathbf{u} \mapsto E(\mathbf{u}) \in B\left(H^{s} \otimes H^{s}, H^{s_{*}} \otimes H^{s_{*}}\right)$ is Lipschitz continuous in the norm topology for $1 \leq s_{*} \leq s-1$. The Lipschitz constant is proportional to a simple algebraic function of the radius $r$ of $W$. This is a standard result; its verification begins with the multiplier property of the partial derivative term acting on the target space for the designated 
indices. One then estimates the target norm of the difference of the matrix entries in the matrix multiplier $A_{2}(\mathbf{u})=\tilde{A}_{0}^{-1}(\mathbf{u}) A_{1}(\mathbf{u})$. The choice $s_{*}>3 / 2$ gives the desired result. The analysis of the linear second order term is immediate. The Lipschitz property follows for the consolidated operator.

The hypotheses on $F$ are directly verified, given the linear dependence on $n, v$, once the smoothing property of the operator $\Phi_{x}$ is noted. In particular, $\Phi_{x}$ is bounded on both $X$ and $Y$. The remaining property to verify is the similarity relation contained in the isomorphism hypothesis (4). This is a consequence of Kato's commutator relation. Indeed, it was shown in [7, appendix] that the commutator $\left[\Gamma^{s} \otimes \Gamma^{s}, A_{2}\right]$ is bounded on $L_{2}(\mathbf{R}) \otimes L_{2}(\mathbf{R})$, if the operator $A_{2}$ is interpreted now as extended to a subspace of $H_{0}^{s}(\mathbf{R}) \otimes H_{0}^{s}(\mathbf{R})$, via the Calderón extension theorem. As shown by Kato, the bound does not exceed a constant times the $C^{1}$ norm of the coefficients of $A_{2}$. This is satisfied if $s>5 / 2$ (note the presence of derivatives in $A_{2}$ ). Since the isomorphism $S$ constructed earlier can be interpreted as (the restriction) of $\Gamma^{s} \otimes \Gamma^{s}$, plus a finite rank

interpolation operator on the null space of $\left(I-d^{2} / d x^{2}\right) \otimes\left(I-d^{2} / d x^{2}\right)$, we may infer the validity of the final condition (4). One has now verified the hypotheses of the general result; if $T$ is sufficiently small, the uniform norm of $n$ is not smaller than $\alpha^{\prime}$; in particular, $A_{0}$ coincides with $\tilde{A}_{0}$. The convergence as $\varepsilon \rightarrow 0$ has appeared in a number of publications (see, for example, [6]). The proof is complete.

\section{Expanding Invariant Regions}

The following result is quoted in [2] as a generalization of a result of Smoller [9, Theorem 14.7]. The bounds of this section apply to the solution derived in Theorem 2.1.

Lemma 3.1 (Fang-Ito) Given a system of nonlinear diffusion equations,

$$
u_{t}=\varepsilon u_{x x}+M(u, x) u_{x}+f(u, x, t), \quad x \in(0,1),
$$

with initial and boundary conditions specified. Let

$$
G_{j}: \mathbf{R}^{N} \mapsto \mathbf{R}, \quad j=1, \ldots, J
$$

be smooth functions, and let $\omega$ and $A$ be two constants. Define

$$
\Sigma(t)=\cap_{j=1}^{J}\left\{u \in \mathbf{R}^{N}: G_{j}(u) \leq A \exp (\omega t)\right\} .
$$

Suppose for $\left(t_{0}, u_{0}\right)$ such that $u_{0} \in \partial \Sigma\left(t_{0}\right)$, i.e.,

$$
G_{j}\left(u_{0}\right)=A \exp \left(\omega t_{0}\right) \text { for some } j
$$


the following conditions hold:

(1)

$$
\nabla G_{j} \text { at } u_{0}
$$

is a left eigenvector of $M\left(u_{0}, x\right) \forall x \in(0,1)$;

(2) $G_{j}$ is quasi-convex at $u_{0}$, i.e., whenever $\nabla G_{j}$ is orthogonal to $r$, then the Hessian quadratic form is nonnegative when evaluated at $r$ :

$$
\nabla^{2} G_{j}(r, r) \geq 0
$$

$$
\nabla G_{j} \cdot f<\omega A \exp \left(\omega t_{0}\right)
$$

Then the trajectory satisfies $u(t) \in \Sigma(t) \forall 0<t \leq T$.

Write the equations $(4,5)$ as:

$$
u_{t}=\varepsilon u_{x x}+M(u, x) u_{x}+f(u, x, t), \quad x \in(0,1),
$$

where

$$
\begin{gathered}
M(u, x)=\left[\begin{array}{cc}
0 & -\frac{1}{m} \\
\frac{p^{2}}{m n^{2}}-k T_{0} & -\frac{2 p}{m n}
\end{array}\right], \\
u(x, t)=\left[\begin{array}{l}
n(x, t) \\
p(x, t)
\end{array}\right],
\end{gathered}
$$

and

$$
f(u, x, t)=\left[\begin{array}{c}
0 \\
-e n \Phi_{x}-\frac{p}{\tau_{p}}
\end{array}\right]
$$

Proposition 3.1 If a solution of the smoothed Euler-Poisson system exists with $n(x, t) \geq \alpha>0$, then $u$ as just defined satisfies the hypotheses of the preceding Fang-Ito lemma with $N=2$ and

$$
G_{1}(n, p)=-\frac{p}{n}+m c \log (n), \quad G_{2}(n, p)=\frac{p}{n}+m c \log (n), c=\sqrt{\frac{k T_{0}}{m}}
$$

and

$$
\omega=\frac{e^{2}}{\epsilon_{0}} \int_{0}^{1}\left(n(x, 0)+\left|n_{D}(x)\right|\right) d x, A=1 .
$$

Proof The eigenvalues of $M$ are determined as

$$
\lambda_{1}=-\frac{p}{m n}+c, \lambda_{2}=-\frac{p}{m n}-c .
$$


Corresponding left eigenvectors are:

$$
\xi^{1}=\left[\begin{array}{ll}
\frac{p}{n^{2}}+\frac{m c}{n} & -\frac{1}{n}
\end{array}\right], \quad \xi^{2}=\left[\begin{array}{ll}
-\frac{p}{n^{2}}+\frac{m c}{n} & \frac{1}{n}
\end{array}\right] .
$$

By direct computation, it is seen that

$$
\nabla G_{1}=\xi^{1}, \quad \nabla G_{2}=\xi^{2} .
$$

Thus, (1) of the lemma holds. To verify (2), suppose that orthogonality holds for $j=1$, so that

$$
r_{1}\left(-\frac{p}{n^{2}}-\frac{m c}{n}\right)+r_{2}\left(\frac{1}{n}\right)=0
$$

for a vector $r$. The quadratic form induced by the Hessian of $G_{1}$ is just

$$
\nabla^{2} G_{1}(r, r)=-\left(\frac{2 p}{n^{3}}+\frac{m c}{n^{2}}\right) r_{1}^{2}+\left(\frac{2}{n^{2}}\right) r_{1} r_{2}=\frac{m c}{n^{2} r_{1}^{2}} \geq 0
$$

Here, we have made use of the orthogonality. The calculation for $G_{2}$ leads to

$$
\nabla^{2} G_{2}(r, r)=\left(\frac{2 p}{n^{3}}-\frac{m c}{n^{2}}\right) r_{1}^{2}-\left(\frac{2}{n^{2}}\right) r_{1} r_{2}=\frac{m c}{n^{2} r_{1}^{2}} \geq 0 .
$$

It follows that (ii) holds. We now consider (iii). Suppose that

$$
G_{1}\left(u_{0}\right)=\exp \left(\omega t_{0}\right), G_{2}\left(u_{0}\right) \leq \exp \left(\omega t_{0}\right) .
$$

In particular, $\frac{p}{n} \leq 0$, which follows from subtracting $G_{2}$ from $G_{1}$. Since

$$
\nabla G_{1} \cdot f=-e \Phi_{x}+\frac{p}{n \tau_{p}}
$$

we are led to obtain a gradient estimate for $\Phi$. Specifically, we know that

$$
\nabla G_{1} \cdot f \leq-e \Phi_{x}
$$

If the Green's function is employed, one obtains

$$
\Phi_{x}=\frac{e}{\epsilon_{0}} \int_{0}^{1} G_{x}(x, \xi)\left(n+n_{D}\right) d \xi
$$

where the Green's function is given by

$$
G(x, \xi)=\left[\begin{array}{cc}
(1-x) \xi, & 0 \leq \xi \leq x \leq 1 \\
(1-\xi) x, & 0 \leq x \leq \xi \leq 1
\end{array}\right]
$$

Since the derivative of the Green's function has an upper bound of unity, we deduce that

$$
\left|\Phi_{x}\right| \leq \frac{e}{\epsilon_{0}} \int_{0}^{1}\left(n+\left|n_{D}\right|\right) d \xi .
$$


In order to estimate $\int_{0}^{1} n(x, t) d x$, we use (4):

$\int_{0}^{1} n(x, t) d x=\int_{0}^{1} n(x, 0) d x-\int_{0}^{t}\left\{n v(1, s)-n v(0, s)-\varepsilon\left[(n)_{x}(1, s)-(n)_{x}(0, s)\right]\right\} d s$.

From the boundary conditions, it follows that

$$
\int_{0}^{1} n(x, t) d x=\int_{0}^{1} n(x, 0) d x .
$$

When coupled with the inequality for $\Phi_{x}$, we conclude that

$$
\left|\Phi_{x}\right| \leq \frac{e}{\epsilon_{0}} \int_{0}^{1}\left(n(x, 0)+\left|n_{D}(x)\right|\right) d x .
$$

The argument in the case, $G_{1} \leq \exp \left(\omega t_{0}\right), G_{2}=\exp \left(\omega t_{0}\right)$, is similar. This concludes the proof.

\section{References}

[1] G.-Q. Chen, J.W. Jerome, C.-W. Shu, and D. Wang. Two -carrier semiconductor device models with geometric structure and symmetry properties. In, Modelling and Computation for Applications in Mathematics, Science, and Engineering, Oxford Univ. Press, 1998, pp. 103-140.

[2] W. Fang and K. Ito. Weak solutions to a one-dimensional hydrodynamic model of two carrier types for semiconductors. Nonlinear Anal. 28 (1997), 947-963.

[3] J. Glimm. Solutions in the large for nonlinear hyperbolic systems of equations. Comm. Pure Appl. Math. 18 (1965), 697-715.

[4] T.R. Hughes, T. Kato, J.E. Marsden. Well-posed quasi-linear second-order hyperbolic systems with applications to nonlinear elastodynamics and general relativity. Arch. Rational Mech. Anal. 63 (1976), 273-294.

[5] J.W. Jerome. Approximation of Nonlinear Evolution Systems, Academic Press, 1983.

[6] J.W. Jerome. An analytical study of smooth solutions of the Blotekjaer hydrodynamic model of electron transport. VLSI Design 15 (2002), 729-742.

[7] T. Kato. Quasi-linear equations of evolution, with applications to partial differential equations. In, Spectral Theory and Differential Equations (W. Everitt, ed.), Springer Lecture Notes 448, 1975, pp. 25-70.

[8] F. Poupaud, M. Rascle, and J.-P. Vila. Global solutions to the isothermal EulerPoisson system with arbitrarily large data. J. Differential Equations 123 (1995), 93-121.

[9] J.Smoller. Shock Waves and Reaction-Diffusion Equations (second ed.) Springer-Verlag (1994) 